\title{
Prevalência de Sintomas de Ansiedade e Depressão em Estudantes de Medicina
}

\author{
Prevalence of Anxiety and Depression \\ Symptoms among Medicine Students
}

\author{
Tatheane Couto de Vasconcelos ${ }^{1}$ \\ Bruno Rafael Tavares Dias ${ }^{I}$ \\ Larissa Rocha Andrade \\ Gabriela Figueirôa Melo ${ }^{I}$ \\ Leopoldo Barbosa ${ }^{I I}$ \\ Edvaldo Souza $a^{I I}$
}

\section{PALAVRAS-CHAVE \\ - Depressão; \\ - Ansiedade; \\ - Estudantes de Medicina; \\ - Educação Médica.}

\section{KEYWORDS}

- Depression;

- Anxiety;

- Medicine Students;

- Medical Education.
Reencaminhado em: 08/09/2014

Aprovado em: 17/10/2014

\begin{abstract}
Objectives: To determine the prevalence of symptoms of anxiety and depression in medical students and evaluate associated factors. Methods: Cross-sectional study involving 234 students, who answered an online questionnaire containing sociodemographic factors and the Hospital Anxiety and Depression Scale (HADS). Results: The mean score of the Hospital Anxiety and Depression Scale for anxiety was 6.7 (+/-3.4), while 34.3\% (80) of the students presented false-positive symptoms of anxiety and $19.7 \%$ (46) suggestive symptoms. In terms of depression the mean HADS score was 4.4 $(+/-3.1)$, with $19.3 \%$ (45) of the students presenting false-positive symptoms of depression and $5.6 \%$ presenting suggestive symptoms. In the univariate analysis, psychoactive drugs use was associated with symptoms of anxiety, for symptoms of depression, being from the Metropolitan Region of Recife $(R M R)$ was found to be a protective factor, as illicit drugs use was associated to risk. Conclusion: The prevalence of anxiety and depression symptoms, associated to the use of psychoactive and illicit drugs respectively, indicates the need for measures aimed at prevention and early diagnosis.
\end{abstract}




\section{INTRODUÇÃO}

Estima-se que de $15 \%$ a $25 \%$ dos estudantes universitários apresentam algum tipo de transtorno psiquiátrico durante a sua formação acadêmica ${ }^{1}$. Dentre esses transtornos, os depressivos e de ansiedade são os mais frequentes ${ }^{1,2,3}$. Em Dubai, um estudo demonstrou que 28,6\% dos estudantes de Medicina apresentavam depressão e 28,7\% manifestavam ansiedade ${ }^{4}$. Uma pesquisa realizada na Universidade Federal de São Paulo mostrou que 38,2\% dos alunos do curso de Medicina apresentavam sintomas depressivos ${ }^{5}$. Um estudo que envolveu estudantes de Medicina conduzido na Estônia evidenciou alta porcentagem de estudantes com sintomas emocionais, dos quais $21,9 \%$ apresentavam sintomas de ansiedade e 30,6\% tinham sintomas de depressão ${ }^{6}$. Os fatores que podem influenciar a prevalência de ansiedade e depressão nos estudantes de Medicina são a elevada carga horária, grande volume de matérias, maior contato com pacientes portadores de diversas doenças e prognósticos, insegurança em relação ao ingresso no mercado de trabalho, cobrança da sociedade e da instituição de ensino, além da autocobrança típica deste curso ${ }^{7}$.

Uma das dificuldades dos cuidados psiquiátricos para os estudantes de Medicina é o fato de que eles tendem a não procurar ajuda médica para seus problemas. ${ }^{8}$ Estudos demonstraram que, apesar do alto nível de aflição que acomete os estudantes de Medicina, apenas de $8 \%$ a $15 \%$ deles procuram cuidado psiquiátrico durante a sua formação. Além disso, os resultados de outro estudo, realizado na Universidade da Pennsylvania, mostraram que, dos $24 \%$ de seus estudantes que declararam ter problemas depressivos, apenas $22 \%$ procuraram ajuda médica ${ }^{9,10}$. Este fato é justificado por inúmeras razões: falta de tempo, estigma associado à utilização de serviços de saúde mental, custos e medo das consequências em nível curricular. Dentre os que procuram, cerca de $22 \%$ a $40 \%$ apresentam perturbação do humor, geralmente depressão, $0^{9,11}$.

Já é bem estabelecido no meio acadêmico que em pessoas com depressão há uma redução do rendimento da aprendizagem nas tarefas cotidianas, ocorrência de baixa autoestima e insegurança. Além disso, é demonstrada uma reciprocidade negativa entre assertividade e nível de ansiedade, sugerindo que esta pode interferir no comportamento assertivo, tão importante durante a formação profissional, acarretando danos ao conhecimento profissional e ao aprendizado da experiência médica, e podendo culminar, inclusive, no abandono do curso e até em suicídio ${ }^{12,13,14}$.

A ocorrência de distúrbios de humor e ansiedade ainda na graduação, quando não detectada e adequadamente tratada, pode se perpetuar ou agravar durante a residência médica e na atividade profissional ${ }^{15,16}$. Assim, o presente estudo teve o objetivo de determinar a prevalência de ansiedade e depressão nos estudantes de Medicina e possíveis fatores associados.

\section{MÉTODOS}

Foi realizado um estudo observacional com delineamento transversal em graduandos do curso de Medicina da Faculdade Pernambucana de Saúde (FPS) do primeiro ao sexto ano, no primeiro semestre de 2012. Os dados foram coletados no mês de junho, por meio da aplicação de um questionário anônimo online, disponível no portal de acesso restrito aos alunos, contendo variáveis sociodemográficas e educacionais e a Escala Hospitalar de Ansiedade e Depressão. Esse instrumento foi utilizado, inicialmente, para avaliar sintomas de ansiedade e depressão em pacientes de hospitais clínicos não psiquiátricos e posteriormente passou a ser usado em pacientes não interna$\operatorname{dos}^{17}$, assim como em indivíduos sadios ${ }^{18}$. Foi disponibilizado no site da FPS e por meio de um link de acesso restrito a cada estudante após leitura e assinatura do Termo de Consentimento Livre e Esclarecido, aplicado no intervalo dos grupos tutoriais.

As variáveis sociodemográficas estudadas foram idade, sexo, procedência, com quem mora, prática de atividade extracurricular, lazer, religiosidade, grau de satisfação com o curso, uso de álcool e drogas ilícitas, como também uso de drogas psicoativas para tratamento psiquiátrico e/ou medicamentoso para ansiedade e depressão.

Escolheu-se a Ehad para avaliar sintomas sugestivos de ansiedade e depressão devido a sua fácil aplicação, já que possui apenas 14 questões intercaladas de ansiedade e depressão e ainda apresenta boa sensibilidade (70,8\% a $80,6 \%)$ e especificidade $(69,6 \%$ a 90,9\%) quando comparada à Escala de Ansiedade de Beck (EAB) e à Escala de Depressão de Beck (EDB), ambas consideradas padrão-ouro ${ }^{19}$. Essa escala diminui a influência das patologias somáticas, uma vez que não utiliza manifestações clínicas inespecíficas, tais como perda de peso, anorexia, insônia, fadiga, pessimismo sobre o futuro, cefaleia, tontura, entre outros, e/ou sintomas de ansiedade ou depressão relacionados a doenças físicas. Caso haja alguma afecção, os transtornos de humor serão determinados pelos sintomas psicológicos, já que estes sobressaem em relação às manifestações somáticas ${ }^{17}$. Os escores da Ehad variam de 0 a 21 para cada subescala, sendo que os participantes com escores menores que 7 são considerados sem sinais clínicos significativos para ansiedade/depressão, entre 8 e 10 com sintomas possíveis (falso-positivos), e acima de 10, sintomas sugestivos de distúrbio.

Os dados obtidos foram salvos automaticamente numa planilha do Excel. Em seguida, os dados foram importados, e 
a análise dos dados foi efetuada com o programa EPI-Info ${ }^{\mathrm{TM}}$ 3.5.1 para Windows ${ }^{\mathrm{TM}}$ e com o SPSS versão 12. Foram construídas tabelas de distribuição de frequência das variáveis estudadas, calculando-se ainda medianas para as variáveis contínuas de distribuição não normal. Para cálculo de associação entre variáveis categóricas de exposição e desfecho, foi realizada inicialmente a análise univariada, utilizando-se o teste de qui-quadrado ou exato de Fisher, quando indicado, com nível de significância de 95\%.

Após a aprovação pelo Comitê de Ética em Pesquisa com Seres Humanos do Instituto de Medicina Integral (Imip), todos os procedimentos éticos propostos e aprovados foram estritamente seguidos pelos pesquisadores.

\section{RESULTADOS}

Participaram deste estudo 234 estudantes. A idade média dos estudantes foi de 22 anos (DP $=+/-3$ ), 65,8\% (154) do sexo feminino, 65,4\% (153) procedentes da Região Metropolitana do Recife (RMR), 19,6\% (45) de outros municípios de Pernambuco e 14,9\% (34) de outros Estados. Em relação à moradia, $61,1 \%$ (142) moram com os pais, $16,6 \%$ (38) com outros familiares, $11,1 \%$ (26) moram sozinhos, 4,2\% (10) moram em pensionato/hotel, 3,4\% (8) moram com parceiro e $3,4 \%$ (8) moram com outras pessoas. A maioria $-52,1 \%$ - (122) referiu possuir parceiro(a) fixo(a). No quesito religião, 51,7\% (121) responderam que a religião é muito importante em suas vidas, 30,3\% (71) responderam que achavam importante, 6,4\% (15) não achavam importante, 5,9\% (14) responderam que achavam pouco importante, e 5,5\% (13), indiferente. No que diz respeito ao lazer, 18,1\% (42) realizam atividade de lazer muito frequentemente, 43,8\% (102) realizam frequentemente, 30,9\% (72) realizam algumas vezes, 6,2\% (14) raramente realizam, e 0,8\% (2) nunca realizam atividade de lazer. Cerca de 95,3\% (223) dos estudantes estão muito satisfeitos ou satisfeitos com o curso. Por sua vez, quanto ao uso de drogas, 68\% (159) relatam uso de álcool eventualmente, $16,6 \%$ (39) relatam uso de droga ilícita e $11,1 \%$ (26) relatam uso de drogas psicoativas. Os participantes estudam em média 19,3 horas por semana (+/- 10,1), não havendo diferença entre os sexos, e 15,4\% (36) exercem atividade extracurricular remunerada.

Em relação à ansiedade, 26,9\% (63) dos estudantes já haviam realizado tratamento psicológico e 25,6\% (60) já tinham usado algum medicamento para tratar a ansiedade. $\mathrm{O}$ escore médio da Ehad para ansiedade foi de 6,7 (+/-3,4), e 34,3\% (80) dos estudantes apresentaram sintomas falso-positivos, sendo que 19,7\% (46) manifestaram sintomas sugestivos de tal transtorno.
Em se tratando da depressão, 13,3\% (31) já tinham feito tratamento, e 11,5\% (27) já haviam usado algum medicamento para tratar a depressão. O escore médio da Escala Hospitalar de Ansiedade e Depressão para depressão foi de 4,4 (DP +/$3,1)$, e 19,3\% (45) dos estudantes apresentaram sintomas falso-positivos de depressão, sendo que 5,6\% (13) apresentaram sintomas sugestivos para o transtorno.

\begin{tabular}{|c|c|c|c|c|c|c|}
\hline \multicolumn{7}{|c|}{$\begin{array}{c}\text { TABELA } 1 \\
\text { Características sociodemográficas e comportamentais } \\
\text { de estudantes segundo a presença de sintomas } \\
\text { sugestivos de ansiedade }\end{array}$} \\
\hline \multirow{2}{*}{$\begin{array}{c}\text { Variáveis } \\
\text { Sexo }\end{array}$} & \multicolumn{2}{|c|}{ Sim } & \multicolumn{2}{|c|}{ Não } & \multirow{2}{*}{$\mathrm{p}$} & \multirow{2}{*}{$\begin{array}{c}\text { RP } \\
\text { (IC 95\%) }\end{array}$} \\
\hline & n & $\%$ & n & $\%$ & & \\
\hline Masculino & 27 & 33,8 & 52 & 34,0 & & \\
\hline Feminino & 53 & 66,2 & 101 & 66,0 & 0,071 & \\
\hline \multicolumn{7}{|l|}{ Procedência } \\
\hline RMR & 49 & 61,2 & 104 & 68,0 & & \\
\hline Fora da RMR & 31 & 38,8 & 49 & 32,0 & 0,304 & \\
\hline \multicolumn{7}{|c|}{ Mora com os pais } \\
\hline Sim & 50 & 62,5 & 93 & 60,7 & & \\
\hline Não & 30 & 37,5 & 60 & 39,2 & 0,798 & \\
\hline \multicolumn{7}{|l|}{ Parceiro fixo } \\
\hline Sim & 44 & 55,0 & 77 & 50,3 & & \\
\hline Não & 36 & 45,0 & 76 & 49,7 & 0,497 & \\
\hline \multicolumn{7}{|l|}{ Drogas ilícitas } \\
\hline Sim & 13 & 16,6 & 23 & 15,0 & & \\
\hline Não & 65 & 83,3 & 130 & 85,0 & 0,746 & \\
\hline \multicolumn{7}{|c|}{ Drogas psicoativas } \\
\hline Não & 16 & 80,0 & 143 & $\begin{array}{r}6,5 \\
93,4\end{array}$ & 0,001 & $\begin{array}{c}1,99 \\
(1,38-2,87)\end{array}$ \\
\hline \multicolumn{7}{|c|}{ Atividade extracurricular remunerada } \\
\hline Sim & 10 & 12,5 & 26 & 17,0 & & \\
\hline Não & 70 & 87,5 & 127 & 83,0 & 0,367 & \\
\hline
\end{tabular}

$\mathrm{Na}$ análise univariada de fatores potencialmente associados à presença de sintomas de ansiedade, não se evidenciaram diferenças estatisticamente significativas em relação a sexo, procedência, morar com a família, ter parceria fixa, uso de drogas ilícitas e ter atividade extracurricular remu- 
nerada. Contudo, o uso de drogas psicoativas se mostrou associado à presença de sintomas sugestivos de ansiedade ( $p<, 05$, RP: 1,99, IC: 1,38 - 2,87), como demonstrado na Tabela 1. Na análise univariada de fatores potencialmente associados à presença de sintomas de depressão, não se evidenciaram diferenças estatisticamente significativas em relação a sexo, morar com a família, ter parceria fixa, uso de drogas psicoativas e ter atividade extracurricular remunerada. Contudo, o fato de o estudante ser procedente da RMR surgiu como fator de proteção ( $p<, 05$, RP: 0,55, IC: $0,33-0,93)$, e o uso de drogas ilícitas ( $<<, 05, \mathrm{RP} 2,03, \mathrm{IC}$ : $1,18-3,50)$ surgiu como fator de risco, como demonstrado na Tabela 2.

\begin{tabular}{|c|c|c|c|c|c|c|}
\hline $\begin{array}{l}\text { Característ } \\
\text { estudantes }\end{array}$ & soc & $\begin{array}{r}\mathrm{T} \\
\text { demo } \\
\text { a pres } \\
\text { de }\end{array}$ & $\begin{array}{l}\text { gráf } \\
\text { ençá } \\
\text { pres }\end{array}$ & $\begin{array}{l}\text { as e co } \\
\text { le sint } \\
0\end{array}$ & $\begin{array}{l}\text { omport } \\
\text { omas s }\end{array}$ & $\begin{array}{l}\text { amentais de } \\
\text { ugestivos de }\end{array}$ \\
\hline Variáveis & & im & & ão & & \\
\hline Sexo & n & $\%$ & n & $\%$ & & \\
\hline Masculino & 13 & 28,8 & 67 & 35,4 & 0,404 & \\
\hline Feminino & 32 & 71,1 & 122 & 64,5 & & \\
\hline Procedência & & & & & & \\
\hline RMR & 23 & 51,1 & 130 & 68,8 & 0,025 & $\begin{array}{c}0,55 \\
(0,33-0,93)\end{array}$ \\
\hline Fora da RMR & 22 & 48,8 & 59 & 31,2 & & \\
\hline Mora com os & & & & & & \\
\hline Sim & 23 & 51,1 & 120 & 63,5 & 0,125 & \\
\hline Não & 22 & 48,8 & 69 & 36,5 & & \\
\hline Parceiro fixo & & & & & & \\
\hline Sim & 26 & 57,7 & 96 & 50,8 & 0,399 & \\
\hline Não & 19 & 42,2 & 93 & 49,2 & & \\
\hline Drogas ilícita & & & & & & \\
\hline Sim & 13 & 28,8 & 26 & 13,7 & 0,014 & $\begin{array}{c}2,03 \\
(1,18-3,50)\end{array}$ \\
\hline Não & 32 & 71,1 & 163 & 86,2 & & \\
\hline Drogas psicoa & & & & & & \\
\hline Sim & 7 & 15,5 & 19 & 10,0 & 0,291 & \\
\hline Não & 38 & 84,4 & 170 & 89,9 & & \\
\hline Atividade ext & Irricu & ar remu & nerad & & & \\
\hline Sim & 6 & 13,3 & 30 & 15,9 & 0,671 & \\
\hline Não & 39 & 86,6 & 159 & 84,1 & & \\
\hline
\end{tabular}

\section{DISCUSSÃO}

Neste trabalho, foi identificado que 34,3\% (80) dos estudantes apresentaram sintomas falso-positivos para ansiedade (pontuação entre 8 e 10 na Ehad-A), sendo que 19,7\% (46) destes apresentaram sintomas sugestivos de ansiedade (pontuação > 10 na Ehad-A). Um estudo desenvolvido na Lituânia investigou sintomas de ansiedade em 338 estudantes do curso de Medicina, utilizando também a Ehad. Foi identificada prevalência de sintomas de ansiedade em $43 \%$ dos estudantes, não diferenciando, no entanto, os escores da Ehad-A ${ }^{20}$. Na Faculdade de Medicina do ABC paulista, 30,9\% dos graduandos de Medicina apresentaram traços de ansiedade alta, e os demais alunos, 69,1\%, demonstraram ansiedade moderada. Uma comparação fidedigna torna-se difícil, tendo em vista a utilização de diferentes escalas nos estudos ${ }^{13}$. Porém, este estudo nos mostra quão elevados podem ser os achados de sintomas de ansiedade no curso médico, tendo em vista que nenhum estudante da amostra foi classificado como tendo ansiedade leve ou sem sintomas ansiosos.

Avaliando de maneira mais ampla, um estudo realizado no Rio de Janeiro mostrou que, dos 149 alunos nos dois anos de residência, $50 \%$ obtiveram pontuação $>9$ na Ehad-A, o que sugere um aumento da prevalência de sintomas ansiosos a partir da residência. Isto corrobora a importância da prevenção e tratamento precoces da ansiedade nos estudantes de Medicina ${ }^{21}$.

O presente estudo demonstrou que 19,3\% (45) dos estudantes apresentaram sintomas falso-positivos para depressão, sendo que 5,6\% deles manifestaram sintomas sugestivos de depressão. No Estado de Goiás, 26,8\% dos alunos apresentaram sintomas depressivos, sendo que 6,9\% apresentaram sintomas depressivos de moderados a graves, e 19,9\% leves ${ }^{22}$. Vale ressaltar que foi usada a escala de depressão de Beck, que, como citado, possui uma correspondência de boa a muito boa com a Ehad.

No Estado de São Paulo, um estudo realizado com estudantes de Medicina utilizando o IDB constatou que apenas $9,2 \%$ da amostra possuíam sintomas de depressão. Esta baixa prevalência é atribuída ao alto ponto de corte utilizado, fato justificado devido à composição da amostra. Os autores preferiram os termos tristeza ou luto para caracterizar os participantes em detrimento de depressão leve. Porém, expuseram os resultados seguindo o ponto de corte mais utilizado para IDB, encontrando $29,8 \%$ de indivíduos "com sintomas leves ou moderados", $8,1 \%$ "com sintomas moderados ou graves" e $1,1 \%$ "com sintomas graves" ${ }^{23}$. Outro estudo realizado em São Paulo também revelou percentual inferior ao encontrado no 
presente estudo, tendo encontrado apenas 12,2\% dos estudantes com sintomas depressivos. Tais valores se devem ao alto ponto de corte utilizado na IDB, que atribui o termo depressão apenas aos estudantes com pontuação superior a 20 pontos, enquanto em outros estudos que utilizam a mesma escala essa pontuação se refere a depressão grave ${ }^{24}$. Já em Uberlândia, os resultados ultrapassaram muito a média de prevalência dos estudos citados: 79\% dos estudantes avaliados tinham depressão de leve a grave. Vale ressaltar que neste estudo o ponto de corte para IDB foi bastante inferior ao dos estudos acima, pois estudantes com pontuação superior a quatro já eram considerados com depressão leve ${ }^{14}$.

De modo geral, os estudantes universitários, principalmente aqueles que precisam se afastar do núcleo familiar em decorrência da localização da universidade, tornam-se mais expostos a distúrbios psicológicos. Neste estudo foi observado que há maior risco de depressão entre os estudantes procedentes de municípios distantes da universidade e que, consequentemente, estavam afastados do âmbito familiar. Este resultado coincide com o encontrado na literatura, a exemplo de um estudo com alunos de Medicina colombianos que afirma ter ocorrido aumento do risco de depressão à medida que diminuiu a qualidade da relação familiar ${ }^{25}$. Além disso, a literatura descreve que o fato de o estudante dispor de pessoas próximas, com quem possa compartilhar sentimentos, é um elemento importante para retardar ou reter os processos de estresse e burnout ${ }^{26}$.

Quanto a outros fatores, estudos realizados numa universidade privada de Curitiba, na Faculdade de Medicina de São José do Rio Preto e em outras faculdades de São Paulo concluíram que a droga mais usada por estudantes de Medicina é o álcool e que $78 \%$, 86\% e $82 \%$ das amostras, respectivamente, já haviam ingerido bebida alcoólica ao menos uma vez na vida; foi constatado, também, aumento do consumo no decorrer do curso $^{27,28,29}$. Em nossa amostra, 68\% dos estudantes relataram uso dessa substância. Já na Faculdade de Medicina da Universidade Federal da Bahia, um estudo que investigou as drogas mais comuns entre estudantes de Medicina revelou que 56,1\% dos alunos relataram uso de álcool ${ }^{30}$.

Quanto ao uso de drogas ilícitas e drogas psicoativas, obtivemos um percentual de $16,6 \%$ (39) e $11,1 \%$ (26), respectivamente. Entre os medicamentos com potencial para abuso, os mais utilizados são os ansiolíticos $(13,1 \%)$ e as anfetaminas $(10,1 \%)$. De acordo com uma pesquisa realizada na Faculdade de Medicina de São José do Rio Preto, os acadêmicos usam tais medicamentos em virtude do extenso conteúdo curricular a ser estudado, com o intuito de melhorar a atenção e/ou se manter acordados durante mais tempo ${ }^{28}$.
Apesar de não ter sido avaliada em nosso estudo, é importante salientar a associação do tabaco com sintomas sugestivos de ansiedade e depressão. A literatura demonstra que o tabagismo está relacionado diretamente com os transtornos de ansiedade, principalmente no sexo feminino, e também com os transtornos depressivos, especialmente o transtorno depressivo maior ${ }^{31,32}$

Os distúrbios relacionados com a saúde mental dos estudantes de Medicina são frequentes, porém poucos alunos buscam tratamento, normalmente por temerem o estigma associado à procura de ajuda e tratamento nestas situações. Assim, relutam em demonstrar tal vulnerabilidade, mesmo quando dispõem de suporte ${ }^{8}$. Confirmando isto, um estudo realizado em Portugal constatou que $10 \%$ dos estudantes que participaram do estudo haviam realizado tratamento medicamentoso sem prescrição médica para distúrbios relacionados a saúde mental, enquanto $30 \%$ já haviam procurado apoio de profissional de saúde por apresentarem sintomas relacionados com a saúde mental alguma vez na vida ${ }^{33}$. Entretanto, em nosso trabalho, obtivemos uma média superior à desse estudo. Para os sintomas relacionados com ansiedade, foi observado que $26,9 \%$ já haviam realizado tratamento psicológico para esse quadro e que $25,6 \%$ já haviam ingerido algum medicamento. Quanto à depressão, embora o percentual encontrado tenha sido de apenas $13,3 \%$ para os estudantes que realizaram tratamento psicológico - valor abaixo do encontrado em um estudo realizado em Uberlândia (que foi de 24\%) -, encontramos que $11,5 \%$ (27) haviam usado algum medicamento para tratar a depressão, contra 5,5\% desse mesmo estudo ${ }^{14}$.

Em nosso estudo, a maioria dos participantes era do sexo feminino e também apresentou maior proporção de sintomas sugestivos de ansiedade e depressão, seguindo o descrito pela literatura ${ }^{14,23}$. Contudo, neste estudo não foi demonstrada diferença estatisticamente significativa, provavelmente pelo tamanho da população estudada.

Neste trabalho, 52,1\% (122) dos estudantes possuíam parceiro(a) fixo(a) e 47,86\% (112) não tinham, o que difere de outros estudos, como o realizado na Universidade de São Paulo (USP), onde havia mais estudantes sem companheiro(a) ${ }^{23}$. A literatura afirma que problemas conjugais mais frequentemente agravam a situação pelas responsabilidades impostas, gerando situações estressantes e aumentando os riscos de depressão ${ }^{34,35}$. No estudo feito em Uberlândia, porém, não foi observada correlação significante entre estado civil dos estudantes e depressão ${ }^{14}$.

Os resultados do presente estudo não demonstraram diferenças estatisticamente significantes entre o exercício de atividade remunerada pelo estudante e sintomas sugestivos 
de ansiedade e depressão, provavelmente devido ao número de estudantes que exerciam outra atividade. Entretanto, uma pesquisa feita pelo Instituto de Pesquisas Econômicas (IPE) da USP revelou que 30\% dos estudantes de Medicina exercem atividades de trabalho não relacionadas à futura profissão. $\mathrm{O}$ estudante, então, suporta uma excessiva sobrecarga, advinda das pressões acadêmicas e acrescida de atividades extra-acadêmicas, que, a longo prazo, pode representar um comprometimento tanto emocional como somático ${ }^{35}$.

Uma das limitações deste estudo é a pequena amostra da população estudada. A baixa adesão pode ter se dado por falta de interesse dos estudantes em consequência de estarem ocorrendo outras pesquisas no mesmo período em que esta foi aplicada, especialmente no momento da assinatura do TCLE, quando ocorreram maiores perdas entre os graduandos concluintes. Devido a problemas técnicos e operacionais, não avaliamos o período do curso dos estudantes, deixando de fazer a análise semestral dos sintomas de ansiedade e depressão ao longo de todo o curso. Embora o uso do tabaco esteja relacionado com ansiedade e depressão, não incluímos essa variável em nosso estudo, assim como não diferenciamos os tipos de drogas ilícitas e psicoativas mais utilizados na população do estudo.

\section{REFERÊNCIAS}

1. Adewuia AO, Ola BA, Aloba OO, Mapayi BM, Oginni OO. Depression amongst Nigerian university students: prevalence and sociodemographic correlates. Soc Psychiatry Psychiatr Epidemiol [online]. 2006. 41(8) [capturado em: 18 abr 2013]; 674-8. Disponível em: http:/ / www.ncbi.nlm. nih.gov/pubmed/16680408

2. Cavestro JM, Rocha FL. Prevalência de depressão entre estudantes universitários. Journal of Brazilian Psiquiatry [online]. 2006. 55(4) [capturado em:18 ago 2013]; 264-267. Disponível em: http://www.scielo.br/scielo. php?pid=S0047-20852006000400001\&script=sci_arttext

3. Cerchiari EAN, Caetano D, Faccenda O. Prevalência de transtornos mentais menores em estudantes universitários. Estudos de Psicologia [online]. 2005. 10(3) [capturado em: 15 set 2013]; 413-420. Disponível em: http:/ / bases.bireme. $\mathrm{br} /$ cgi-bin/wxislind.exe/iah/online/?IsisScript=iah/iah. xis\&nextAction $=\operatorname{lnk} \&$ base $=$ LILACS\&exprSearch $=443251$ \&indexSearch $=$ ID\&lang $=\mathrm{p}$

4. Ahmed I, Banu H, Al-Fageer R, Al-Suwaidi R. Cognitive emotions: depression and anxiety in medical students and staff. Journal of critical care [online]. 2009. 24(3) [capturado em: 12 jul 2013]; 1-7. Disponível em: http:/ / www.ncbi. nlm.nih.gov/pubmed/19664516
5. Baldassin S, Alves TCL, Andrade AG. The characteristics of depressive symptoms in medical students during medical education and training: a cross-sectional study. BMC Med Educ [online]. 2008. 8(60) [capturado em:12 jul 2013]; http:/ /www.biomedcentral.com/1472-6920/8/60.

6. Eller T, Aluoja A, Vasar V, Veldi M. Symptoms of anxiety and depression in Estonian medical students with sleep problems. Depress Anxiety [online]. 2006. 23(4) [capturado em:18 abr 2013..]; 250-6. Disponível em: http:/ / www.ncbi. nlm.nih.gov/pubmed/16555263

7. Porcu M, Fritzen VC, Helber C. Sintomas depressivos nos estudantes de Medicina da Universidade Estadual de Maringá. Psiquiatria na Prática Médica [online]. 2001. 34(1) [capturado em: 18 ago 2013]. Disponível em: http:/ / www. unifesp.br/dpsiq/polbr/ppm/original5_01.htm.

8. Yiu V. Supporting the well-being of medical students. CMAJ [online].2005. 172(7) [capturado em...]; 889-90. Disponível em: http://www.ncbi.nlm.nih.gov/pmc/articles/PMC554874/

9. Shaw DL, Wedding D, Zeldow PB, Diehl N. Special Problems of Medical Students: Cap 6. In Wedding D, eds. Behavior \& Medicine. Hogrefe Publishing [online]. 2006. [capturado em 18 ago 2013...]; 67-83. Disponível em: http:// www.hogrefe.de/programm/media/catalog/Book/ chapter6.pdf

10. Chew-Graham CA, Rogers A, Yassin N. 'I wouldn't want it on my CV or their records': medical students' experiences of help-seeking for mental health problems. Medical Educcation [online]. 2004. 37(10) [capturado em: 18 ago 2013...]; 878-80. Disponível em: http://www.ncbi.nlm.nih.gov/ pubmed/12974841

11. Gross CP, Mead LA, Ford DE, Klag MJ. Physician, heal thyself? Regular source of care and use of preventive health services among physicians. Arch Intern Med [online]. 2000. 160(21) [capturado em: 12 jul 2013..]; 3209-3214. Disponível em: http://archinte.jamanetwork.com/article. aspx?articleid $=485562$

12. Bandeira M, Quaglia MAC, Bachetti LS, Ferreira TL, Souza GG. Comportamento assertivo e sua relação com ansiedade, lócus de controle e auto-estima em estudantes universitários. Estudos de Psicologia [online]. 2005. 22(2) [capturado em: 15 set 2013];..]; 111-121. Disponível em: http://www.scielo.br/scielo.php?pid=S0103-166X2005000200001\&script=sci_abstract\&tlng=pt

13. Baldassin SP, Martins LC, Andrade AG. Traços de ansiedade entre estudantes de medicina. Arquivos de Medicina-ABC [online]. 2006. 31(1) [capturado em: 12 jul 2013.]; 27-31. Disponível em: http://bases.bireme.br/cgibin/ 
wxislind.exe/iah/online/?IsisScript=iah/iah. $x i s \& s r c=g o$ ogle\&base $=$ LILACS\&lang $=p \&$ nextAction $=\operatorname{lnk} \&$ exprSearc $\mathrm{h}=457920$ \&indexSearch $=\mathrm{ID}$

14. Rezende CHA, Abrão CB, Coelho EP, Passo LBS. Prevalência de Sintomas Depressivos entre Estudantes de Medicina da Universidade Federal de Uberlândia. Rev Bras Educ Med [online]. 2008. 32(3) [capturado em: 15 set 2013];... ; 315-323. Disponível em: http://www.scielo.br/ pdf/rbem/v32n3/v32n3a06.pdf

15. Martins LAN. Atividade médica: fatores de risco para a saúde mental do médico. Rev Bras Clin Terap 1991; 20(9): 355-64

16. Meleiro AMAS. Suicídio entre médicos e estudantes de medicina. Rev Assoc Med Bras [online]. 1998. 44(2) [capturado em: 18 ago 2013.]; 135-140. Disponível em: http:/ / www.scielo.br/scielo.php?script=sci_arttext\&pid $=$ S0104-42301998000200012

17. Botega NJ, Bio MR, Zomignani MA. Transtornos de humor em enfermarias de clínica médica e validação de escala de medida (HAD) de ansiedade e depressão. Rev Saúde Pública [online]. 1995. 29(5) [capturado em: 18 ago 2013]; 355-363. Disponível em: http://www.scielo.br/pdf/rsp/ v29n5/04.pdf

18. Andrews B, Hejdenberg J, Wilding J. Student anxiety and depression: Comparison of questionnaire and interview assessments. J Affect Disord [online]. 2006. 95(1-3) [capturado em: 18 ago 2013.]; 29-34. Disponível em: http:// www.ncbi.nlm.nih.gov/pubmed/16764939

19. Marcolino JAM, Mathias LAST, Piccinini FL. Escala hospitalar de ansiedade e depressão: estudo da validade de critério e da confiabilidade com pacientes no pré-operatório. Rev Bras Anestesiol [online]. 2007. 57(1) [capturado em: 18 ago 2013 ]; 52-62. Disponível em: http:/ /www.scielo.br/ scielo.php?pid=S0034-70942007000100006\&script $=$ sci arttext

20. Bunevicius A, Katkute A, Bunevicius R. Symptoms of anxiety and depression in medical students and in humanities students: relationship with big-five personality dimensions and vulnerability to stress. Int J Soc Psychiatry [online]. 2008. 54(6) [capturado em: 18 ago 2013..]; 494501. Disponível em: http://www.ncbi.nlm.nih.gov/pubmed/18974188

21. Silva GCC, Koch HA, Souza EG. Ansiedade e depressão em residentes em Radiologia e Diagnóstico por Imagem. Rev Bras Educ Med [online]. 2010. 34(2) [capturado em: 18 abr 2013 ]; 199-206. Disponível em: http:// www.scielo.br/scielo.php?script=sci_arttext\&pid $=$ S0100-55022010000200003
22. Amaral GF, Gomide LMP, Batista MP. Sintomas depressivos em acadêmicos de medicina da Universidade Federal de Goiás: um estudo de prevalência. Rev psiquiatr Rio Gd Sul [online]. 2008.30(2) [capturado em: 18 abr 2013]; 124-130. Disponível em: http://www.scielo.br/scielo.php?pid=S0101$-81082008000300008 \&$ script $=$ sci_abstract\&tlng $=p t$

23. Souza, L. Prevalência de sintomas depressivos, ansiosos e estresse em acadêmicos de medicina. São Paulo; 2010. Doutorado [Tese] - Faculdade de Medicina da Universidade de São Paulo.

24. Vallilo NG, Júnior RD, Gobbo R. Prevalência de sintomas depressivos em estudantes de medicina. Ver Bras Clin Med [online]. 2011. 9(1) [capturado em: 18 abr 2013..]; 36-41. Disponível em: http://files.bvs.br/ upload/S/1679-1010/2011/v9n1/a1720.pdf

25. Gaviria S, Rodriguez MA, Alvarez T. Calidad de la relación familiar y depresión en estudiantes de Medicina de Medellín. Rev Chil Neuro-Psiquiatr [online]. 2002. 40(1) [capturado em: 18 abr 2013]; 41-46. Disponível em: http://www.scielo.cl/scielo.php?pid=S0717-92272002000100005\&script=sci_arttext

26. Benevides-Pereira AMT. O processo de adoecer pelo trabalho. São Paulo: Casa do Psicólogo; 2002. p.105-132.

27. Tockus D, Gonçalves PS. Detecção do uso de drogas de abuso por estudantes de medicina de uma universidade privada. J Bras Psiquiatr [online]. 2008. 57(3) [capturado em: 18 abr 2013], 184-187. Disponível em: http:/ /www.scielo. br/scielo.php?pid=S0047-20852008000300005\&script=sci_ abstract\&tlng=pt.

28. Pinton FA, Boskovitz EP, Cabrera EMS. Uso de drogas entre os estudantes de medicina da faculdade de medicina de São José do Rio Preto. Arq Cienc Saúde [online]. 2002. 12(2) [capturado em: 15 set 2013];]; 91-96. Disponível: http:/ /bases.bireme.br/cgi-bin/wxislind.exe/iah/online/?IsisScript=iah/ iah. $x$ is\&src $=$ google\&base $=$ LILACS\&lang $=$ \&\&nextAction $=1$ nk\&exprSearch $=431141$ \&indexSearch $=$ ID

29. Magalhães MP, Barros RS, Silva MTA. Uso de drogas entre universitários: a experiência com maconha como fator delimitante. Rev ABP-APAL [online]. 1991. 13\{3\} [capturado em: 18 abr 2013 ]; 97-104. Disponível: http:/ /bases.bireme. $\mathrm{br} /$ cgi-bin/wxislind.exe/iah/online/?IsisScript=iah/iah. xis\&src $=$ google $\&$ base $=$ LILACS\&lang $=p \&$ nextAction $=\operatorname{lnk}$ \&exprSearch $=123230$ \&indexSearch $=$ ID

30. Almeida AM, Godinho TM, Bitencourt GV. Common mental disorders among medical students. J Bras Psiquiatr [online]. 2007. 56(4) [capturado em: 18 abr 2013]; 245-251. Disponível em: http:/ /www.scielo.br/scielo.php?script=sci arttext\&pid=S0047-20852007000400002 
31. Munaretti CL, Terra MB. Transtornos de ansiedade: um estudo de prevalência e comorbidade com tabagismo em um ambulatório de psiquiatria. J Bras Psiquiatr [online]. 2007. 56(2) [capturado em: 18 abr 2013 ]; 108-15. Disponível em: http://www.scielo.br/scielo.php?pid=S0047-20852007000200006\&script=sci_arttext

32. Rondina RC, Gorayeb R, Botelho C. Psychological characteristics associated with tobacco smoking behavior. J Bras Pneumol [online]. 2007. 33(5) [capturado em: 18 abr 2013]; 592-601. Disponível em: http://www.scielo.br/ scielo.php?pid=S1806-37132007000500016\&script=sci_ arttext\&tlng=en

33. Roberto AR. A Saúde Mental dos Estudantes de Medicina da Universidade da Beira Interior. Covilhã; 2009. Mestrado [Dissertação] - Universidade da Beira Interior Faculdade de Ciências da Saúde.

34. Ferreira RA, Peret Filho RA, Goulart EMA. O estudante de medicina da Universidade Federal de Minas Gerais: perfil e tendências. Rev Assoc Med Bras [online]. 2000. 46(3) [capturado em: 15 set 2013];]; 224-231. Disponível em: http:/ / www.scielo.br/scielo.php?script=sci_arttext\&pid =S0104-42302000000300007
35. Fortes JRA. Saúde mental do universitário. Neurobiologia.1973; 36 (suplemento): 13-24.

\section{CONTRIBUIÇÃO DOS AUTORES}

Tatheane Couto de Vasconcelos, Bruno Rafael Tavares Dias, Larissa Rocha Andrade, Gabriela Figueirôa Melo, Leopoldo Barbosa - planejamento, coleta e análise dos dados.

\section{CONFLITO DE INTERESSES}

Declarou não haver.

\section{ENDEREÇO PARA CORRESPONDÊNCIA}

Edvaldo Souza

Av. Jean Emile Favre, 422

Imbiribeira - Recife

CEP 51200-060 - PE

E-mail: essouza@terra.com.br 